\title{
A minimally-intrusive fully 3D separated plate formulation in computational structural mechanics
}

\author{
Giacomo Quaranta ${ }^{1}$, Mustapha Ziane ${ }^{1}$, Eberhard Haug ${ }^{1}$, Jean-Louis Duval ${ }^{1}$ \\ and Francisco Chinesta ${ }^{2 *}$
}

${ }^{*}$ Correspondence:

Francisco.CHINESTA@ensam.eu

${ }^{2}$ PIMM, ENSAM ParisTech \& ESI

GROUP Chair on Advanced

Modeling and Simulation of

Manufacturing Processes, 151

Boulevard de l'Hopital, 75013

Paris, France

Full list of author information is

available at the end of the article

\begin{abstract}
Most of mechanical systems and complex structures exhibit plate and shell components. Therefore, 2D simulation, based on plate and shell theory, appears as an appealing choice in structural analysis as it allows reducing the computational complexity. Nevertheless, this 2D framework fails for capturing rich physics compromising the usual hypotheses considered when deriving standard plate and shell theories. To circumvent, or at least alleviate this issue, authors proposed in their former works an in-plane-out-of-plane separated representation able to capture rich 3D behaviors while keeping the computational complexity the one of 2D simulations. In the present paper we propose an efficient integration of fully 3D descriptions into existing plate software.
\end{abstract}

Keywords: Plate and Shells theories, In-plane-out-of-plane separated representations, PGD, Dynamics

\section{Introduction}

We consider the linear elastostatic problem defined in the plate domain $\Omega=\Omega_{x y} \times \Omega_{z}$, with $\Omega_{x y}=\left[0, H_{x}\right] \times\left[0, H_{y}\right]$ and $\Omega_{z}=\left[0, H_{z}\right]$ in which the thickness (out-of-plane) dimension is much lower than the other ones, i.e. $H_{z} \ll H_{x}, H_{y}$.

The linear elastic behavior relating the Cauchy's stress $\sigma$ and the strain $\varepsilon$ using Voigt notation reads

$$
\sigma=\mathrm{C} \varepsilon
$$

where $\mathbf{C}$ is the elasticity matrix. The relation between strain $\varepsilon$ and displacement $\mathbf{u}$ (with components $\mathbf{u}=(u, v, w))$ writes

$$
\boldsymbol{\varepsilon}=\nabla_{s} \mathbf{u}=\mathbf{G u},
$$

where $\mathbf{G}=\nabla_{s} \bullet=\frac{1}{2}\left(\nabla \bullet+\nabla^{T} \bullet\right)$ is the symmetric gradient operator. 
Considering an homogeneous and isotropic material the behavior writes

$$
\mathbf{C}=\frac{E}{(1+v)(1-2 v)}\left[\begin{array}{cccccc}
1-v & v & v & 0 & 0 & 0 \\
v & 1-v & v & 0 & 0 & 0 \\
v & v & 1-v & 0 & 0 & 0 \\
0 & 0 & 0 & \frac{(1-2 v)}{2} & 0 & 0 \\
0 & 0 & 0 & 0 & \frac{(1-2 v)}{2} & 0 \\
0 & 0 & 0 & 0 & 0 & \frac{(1-2 v)}{2}
\end{array}\right] \text {. }
$$

With $\mathbf{g}(\mathbf{x})$ the body forces, the displacement field $\mathbf{u}(\mathbf{x})$ for $\mathbf{x} \in \Omega$ is described by the linear momentum balance equation

$$
\nabla \cdot \boldsymbol{\sigma}+\mathbf{g}=\mathbf{0}
$$

The domain boundary $\partial \Omega$ is partitioned into Dirichlet, $\Gamma_{D}$, and Neumann, $\Gamma_{N}$, boundaries, where displacement $\mathbf{u}_{g}$ and tractions $\mathbf{T}$ are enforced respectively. In what follows and without loss of generality we assume $\mathbf{T}=\mathbf{0}$

The problem weak form associated to the strong form (4) lies in looking for the displacement field $\mathbf{u}$ verifying the Dirichlet boundary conditions such that the weak form

$$
\int_{\Omega} \boldsymbol{\varepsilon}\left(\mathbf{u}^{*}\right) \cdot(\mathbf{C} \cdot \boldsymbol{\varepsilon}(\mathbf{u})) d \mathbf{x}=\int_{\Omega} \mathbf{u}^{*} \cdot \mathbf{g} d \mathbf{x},
$$

fulfills for any test function $\mathbf{u}^{*}$, with the trial and test fields defined in appropriate functional spaces.

\section{Plate theory}

The Reissner-Mindlin theory is based on the following fundamental hypotheses [1]: (i) on the middle plane $(z=0)$ the in-plane displacements vanish, i.e. $u(x, y, z=0)=v(x, y, z=$ $0)=0$ that implies that points located in the middle-plane only moves vertically; (ii) the plate thickness remains unchanged; (iii) the plane stress assumption remains valid, i.e. $\sigma_{z z}=0$ and (v) a straight line normal to the undeformed middle plane remains straight but not necessarily orthogonal to the middle plane after deformation.

From these assumptions the displacement field can be written as:

$$
\left\{\begin{array}{l}
u(x, y, z)=-z \theta^{x}(x, y) \\
v(x, y, z)=-z \theta^{y}(x, y) \\
w(x, y, z)=w(x, y)
\end{array}\right.
$$

where $w$ is the vertical displacement (deflection) of the points on the middle plane and the rotations $\theta_{x}$ and $\theta_{y}$ coincide with the angles followed by the normal vectors contained in the planes $x z$ and $y z$ respectively in their motions.

We define the generalized displacement vector $\hat{\mathbf{u}}$

$$
\hat{\mathbf{u}}=\left[\theta^{x}, \theta^{y}, w\right]^{T}
$$

defined at any point on the middle plane.

Injecting plate theory assumptions into the $3 \mathrm{D}$ elastostatic problem weak form, Eq. (5) reduces to the following $2 \mathrm{D}$ formulation

$$
\int_{\Omega_{x y}} \hat{\boldsymbol{\varepsilon}}\left(\hat{\mathbf{u}}^{*}\right) \cdot(\hat{\mathbf{C}} \hat{\boldsymbol{\varepsilon}}(\hat{\mathbf{u}})) d \mathbf{x}=\int_{\Omega_{x y}} \hat{\mathbf{u}}^{*} \cdot \hat{\mathbf{g}} d \mathbf{x},
$$


whose standard finite element discretization leads to

$$
\mathbf{K}_{x y} \mathbf{U}=\mathbf{B}_{x y}
$$

where for notational simplicity the hat symbol $(\hat{\bullet})$ is omitted. In the previous expression (9), $\mathbf{K}_{x y}$ is the stiffness matrix and $\mathbf{U}$ and $\mathbf{B}_{x y}$ are the vector of the generalized displacements and forces, the former containing nodal rotations and deflections and the last the dual quantities: the nodal moments and vertical nodal forces. The 3D displacement field can be then recovered by using the relations (6).

In many cases, the complexity of the solution makes impossible the introduction of pertinent hypotheses for reducing the dimensionality of the model from 3D to $2 \mathrm{D}$. In that case a fully $3 \mathrm{D}$ descriptions seem compulsory, and the in-plane-out-of-plane separated representations become particularly suitable.

\section{PGD-based in-plane-out-of-plane decomposition}

The in-plane-out-of-plane separated representation was applied in our former works to efficiently solve 3D elastic problems in plate geometries [2-4]. The elastic problem was defined in a plate domain $\Omega=\Omega_{x y} \times \Omega_{z}$ with $(x, y) \in \Omega_{x y}, \Omega_{x y} \subset \mathbb{R}^{2}$ and $z \in \Omega_{z}$, $\Omega_{z} \subset \mathbb{R}$. The separated representation of the displacement field $\mathbf{u}=(u, v, w)$ consists in a finite sum decomposition on $N$ terms, each one of them consisting in the product of two unknown functions, one depending on the in-plane coordinates $(x, y)$ and one on the out-of-plane coordinate $z$, i.e.:

$$
\mathbf{u}(x, y, z)=\left(\begin{array}{c}
u(x, y, z) \\
v(x, y, z) \\
w(x, y, z)
\end{array}\right) \approx \sum_{i=1}^{N}\left(\begin{array}{c}
u_{x y}^{i}(x, y) \cdot u_{z}^{i}(z) \\
v_{x y}^{i}(x, y) \cdot v_{z}^{i}(z) \\
w_{x y}^{i}(x, y) \cdot w_{z}^{i}(z)
\end{array}\right) .
$$

Expression (10) can be written in a more compact form by using the Hadamard (component-to-component) product:

$$
\mathbf{u}(x, y, z) \approx \sum_{i=1}^{N} \mathbf{U}_{x y}^{i}(x, y) \circ \mathbf{U}_{z}^{i}(z) .
$$

\section{Enriched formulations}

As reported in the previous section plate kinematics can be written as a single-term separated decomposition

$$
\left\{\begin{array}{l}
u(x, y, z)=\theta^{x}(x, y) f^{x}(z) \\
v(x, y, z)=\theta^{y}(x, y) f^{y}(z) \\
w(x, y, z)=w(x, y) f^{z}(z)
\end{array}\right.
$$

with $f^{x}(z)=-z, f^{y}(z)=-z$ and $f^{z}(z)=1$.

For the sake of generality we are considering generic functions $f^{x}(z), f^{y}(z)$ and $f^{z}(z)$ assumed known, but than can be different to the ones related to the standard ReissnerMindlin plate theory, and its associated 3D kinematics given by Eq. (12). Consequently $\theta^{x}, \theta^{y}$ and $w$ do not represent rotations and deflection anymore. 
The displacements gradient becomes

$$
\nabla \mathbf{u}=\left(\begin{array}{c}
\frac{\partial u}{\partial x} \\
\frac{\partial u}{\partial y} \\
\frac{\partial u}{\partial z} \\
\frac{\partial v}{\partial x} \\
\frac{\partial v}{\partial y} \\
\frac{\partial v}{\partial z} \\
\frac{\partial w}{\partial x} \\
\frac{\partial w}{\partial y} \\
\frac{\partial w}{\partial z}
\end{array}\right)=\left(\begin{array}{c}
\frac{\partial \theta^{x}}{\partial x} \\
\frac{\partial \theta^{x}}{\partial y} \\
\theta^{x} \\
\frac{\partial \theta^{y}}{\partial x} \\
\frac{\partial \theta^{y}}{\partial y} \\
\theta^{y} \\
\frac{\partial w}{\partial x} \\
\frac{\partial w}{\partial x} \\
w
\end{array}\right) \circ\left(\begin{array}{c}
f^{x} \\
f^{x} \\
\frac{d f^{x}(z)}{d z} \\
f^{y} \\
f^{y} \\
\frac{d f^{y}(z)}{d z} \\
f^{z} \\
f^{z} \\
\frac{d f^{z}}{\partial z}
\end{array}\right),
$$

that allows defining the strain separated form, that taking into account its symmetry reads

$$
\begin{aligned}
\boldsymbol{\varepsilon} & =\left(\begin{array}{c}
\frac{\partial u}{\partial x} \\
\frac{\partial v}{\partial y} \\
\frac{\partial w}{\partial z} \\
\frac{\partial u}{\partial y}+\frac{\partial v}{\partial x} \\
\frac{\partial u}{\partial z}+\frac{\partial w}{\partial x} \\
\frac{\partial v}{\partial z}+\frac{\partial w}{\partial y}
\end{array}\right)=\left(\begin{array}{c}
\frac{\partial \theta^{x}}{\partial x} \\
\frac{\partial \theta^{y}}{\partial y} \\
w \\
\frac{\partial \theta^{x}}{\partial y} \\
\theta^{x} \\
\theta^{y}
\end{array}\right) \circ\left(\begin{array}{c}
f^{x} \\
f^{y} \\
\frac{d f^{z}}{d z} \\
f^{x} \\
\frac{d f^{x}}{d z} \\
\frac{d f^{y}}{d z}
\end{array}\right)+\left(\begin{array}{c}
0 \\
0 \\
0 \\
\frac{\partial \theta^{y}}{\partial x} \\
\frac{\partial w}{\partial x} \\
\frac{\partial w}{\partial y}
\end{array}\right) \circ\left(\begin{array}{c}
0 \\
0 \\
0 \\
f^{y} \\
f^{z} \\
f^{z}
\end{array}\right) \\
& =\boldsymbol{\Theta}^{1}(x, y) \circ \mathbf{F}^{1}(z)+\mathbf{\Theta}^{2}(x, y) \circ \mathbf{F}^{2}(z) .
\end{aligned}
$$

In the case of a general material the Hooke tensor can also be written as

$$
\mathbf{C}(x, y, z)=\sum_{i=1}^{M} \mathbf{C}_{x y}^{i}(x, y) \circ \mathbf{C}_{z}^{i}(z) .
$$

For an homogenous material we have simply

$$
\mathbf{C}=\mathbf{C}_{x y}^{i} \circ \mathbf{C}_{z}^{i}
$$

where $\mathbf{C}_{z}$ is given by Eq. (3) and $\mathbf{C}_{x y}$ is a tensor whose all the entries are 1,

$$
\mathbf{C}_{x y}=\left[\begin{array}{llllll}
1 & 1 & 1 & 1 & 1 & 1 \\
1 & 1 & 1 & 1 & 1 & 1 \\
1 & 1 & 1 & 1 & 1 & 1 \\
1 & 1 & 1 & 1 & 1 & 1 \\
1 & 1 & 1 & 1 & 1 & 1 \\
1 & 1 & 1 & 1 & 1 & 1
\end{array}\right] .
$$

Taking this into consideration the method that we are going to explain can be used both for homogenous and not homogenous materials. For the sake of simplicity we are going to present it in the case where in expression (16) only one term appears in the sum, but it can be easily extended to involve more terms. The virtual work principle, expressed using a matrix notation, involves the internal work

$$
\begin{aligned}
\boldsymbol{\varepsilon}^{* T} \boldsymbol{\sigma} & =\boldsymbol{\varepsilon}^{* T} \mathbf{C} \boldsymbol{\varepsilon} \\
& =\left\{\boldsymbol{\Theta}^{1 *}(x, y) \circ \mathbf{F}^{1}(z)+\boldsymbol{\Theta}^{2 *}(x, y) \circ \mathbf{F}^{2}(z)\right\}^{T}\left\{\mathbf{C}_{x y}(x, y) \circ \mathbf{C}_{z}(z)\right\}
\end{aligned}
$$




$$
\begin{aligned}
& \left\{\boldsymbol{\Theta}^{1}(x, y) \circ \mathbf{F}^{1}(z)+\boldsymbol{\Theta}^{2}(x, y) \circ \mathbf{F}^{2}(z)\right\} \\
& =\boldsymbol{\Theta}^{1 * T}(x, y)\left\{\mathbf{C}_{x y}(x, y) \circ \hat{\mathbf{C}}_{z}^{11}(z)\right\} \boldsymbol{\Theta}^{1}(x, y)+\boldsymbol{\Theta}^{1 * T}(x, y)\left\{\mathbf{C}_{x y}(x, y) \circ \hat{\mathbf{C}}_{z}^{12}(z)\right\} \\
& \boldsymbol{\Theta}^{2}(x, y)+\boldsymbol{\Theta}^{2 * T}(x, y)\left\{\mathbf{C}_{x y}(x, y) \circ \hat{\mathbf{C}}_{z}^{21}(z)\right\} \boldsymbol{\Theta}^{1}(x, y)+\boldsymbol{\Theta}^{2 * T}(x, y) \\
& \left\{\mathbf{C}_{x y}(x, y) \circ \hat{\mathbf{C}}_{z}^{22}(z)\right\} \boldsymbol{\Theta}^{2}(x, y) .
\end{aligned}
$$

In the previous expression matrices $\hat{\mathbf{C}}_{z}^{i j}(z)$ results

$$
\hat{\mathbf{C}}_{z_{k l}}^{i j}(z)=\mathbf{C}_{z_{k l}}(z) \mathbf{F}_{k}^{i}(z) \mathbf{F}_{l}^{j}(z), \quad i, j \in[1,2] \& k, l \in[1, \cdots, 6] .
$$

Now, the virtual work integral reads

$$
\begin{aligned}
& \int_{\Omega_{x y} \times \Omega_{z}} \sum_{i=1}^{2} \sum_{j=1}^{2} \boldsymbol{\Theta}^{i * T}(x, y)\left\{\mathbf{C}_{x y}(x, y) \circ \hat{\mathbf{C}}_{z}^{i j}(z)\right\} \boldsymbol{\Theta}^{j}(x, y) d z d x d y \\
& =\int_{\Omega_{x y}} \sum_{i=1}^{2} \sum_{j=1}^{2} \boldsymbol{\Theta}^{i * T}(x, y) \mathbf{D}^{i j}(x, y) \boldsymbol{\Theta}^{j}(x, y) d x d y,
\end{aligned}
$$

where

$$
\mathbf{D}^{i j}(x, y)=\mathbf{C}_{x y}(x, y) \circ \int_{\Omega_{z}} \hat{\mathbf{C}}_{z}^{i j}(z) d z .
$$

Now, if we assume an approximation based on a piecewise linear interpolation on a triangular finite element, related to an in-plane mesh of $\Omega_{x y}=\cup_{e=1}^{E} \Omega_{x y}^{e}$, with the shape functions defined by $N_{i}^{e}(x, y), i=1,2,3 ; e=1, \ldots, \mathrm{E}$; it results

$$
\left\{\begin{array}{l}
\theta^{x, e}(x, y)=N_{1}^{e}(x, y) \theta_{1}^{x, e}+N_{2}^{e}(x, y) \theta_{2}^{x, e}+N_{3}^{e}(x, y) \theta_{3}^{x, e} \\
\theta^{y, e}(x, y)=N_{1}^{e}(x, y) \theta_{1}^{y, e}+N_{2}^{e}(x, y) \theta_{2}^{y, e}+N_{3}^{e}(x, y) \theta_{3}^{y, e} \\
w^{e}(x, y)=N_{1}^{e}(x, y) w_{1}^{e}+N_{2}^{e}(x, y) w_{2}^{e}+N_{3}^{e}(x, y) w_{3}^{e}
\end{array}\right.
$$

Using that approximation we can express vectors $\Theta^{i}(x, y)$ in each element $\Omega^{e}$ from the generalized nodal displacements

$$
\mathbf{U}^{e T}=\left(\theta_{1}^{x, e}, \theta_{1}^{y, e}, w_{1}^{e}, \theta_{2}^{x, e}, \theta_{2}^{y, e}, w_{2}^{e}, \theta_{3}^{x, e}, \theta_{3}^{y, e}, w_{3}^{e}\right),
$$

from

$$
\boldsymbol{\Theta}^{i}\left((x, y) \in \Omega_{x y}^{e}\right)=\mathbf{B}^{i, e}(x, y) \mathbf{U}^{e},
$$

where $\mathbf{B}^{i, e}(x, y)$ contains the shape functions and theirs derivatives, according to the expressions involved in the components of $\Theta^{i}(x, y), i=1,2$.

Thus, integral (21) reads

$$
\begin{aligned}
& \sum_{e=1}^{E} \mathbf{U}^{e * T}\left\{\int_{\Omega_{x y}^{e}} \sum_{i=1}^{2} \sum_{j=1}^{2} \mathbf{B}^{i, e T}(x, y) \mathbf{D}^{i j}(x, y) \mathbf{B}^{j, e}(x, y) d x d y\right\} \mathbf{U}^{e} \\
& =\sum_{e=1}^{E} \mathbf{U}^{e * T} \mathbf{K}_{x y}^{e} \mathbf{U}^{e}=\mathbf{U}^{* T} \mathbf{K}_{x y} \mathbf{U} .
\end{aligned}
$$

Now, if we consider the virtual work of the body forces $\mathbf{g}(\mathbf{x})$, it involves

$$
\mathbf{u}^{* T} \mathbf{g}(\mathbf{x}) \text {, }
$$

where without loss of generality we assume

$$
\mathbf{u}(x, y, z)=\mathbf{V} \circ \mathbf{W},
$$


with $\mathbf{V}^{T}=\left(\theta^{x}, \theta^{y}, w\right)$ and $\mathbf{W}^{T}=\left(f^{x}(z), f^{y}(z), f^{z}(z)\right)$, and the single-mode decomposition of the body forces given by

$$
\mathbf{g}(x, y, z)=\mathbf{G} \circ \mathbf{H},
$$

with $\mathbf{G}^{T}=\left(M^{x}(x, y), M^{y}(x, y), T(x, y)\right)$ and $\mathbf{H}^{T}=\left(h^{x}(z), h^{y}(z), h^{z}(z)\right)$. The fact of considering a single mode in the decomposition of the body force is not restrictive as discussed later.

The virtual work (27) can be expressed as

$$
\mathbf{u}^{* T} \mathbf{g}(\mathbf{x})=\mathbf{V}^{* T}(x, y) \hat{\mathbf{J}}(z) \mathbf{G}(x, y),
$$

where matrix $\hat{\mathbf{J}}$ reads

$$
\hat{\mathbf{J}}_{k l}(z)=\mathbf{I}_{k l} \mathbf{W}_{k}(z) \mathbf{H}_{l}(z),
$$

with I the identity matrix.

Now, the integral results

$$
\int_{\Omega_{x y} \times \Omega_{z}} \mathbf{u}^{* T} \mathbf{g}(\mathbf{x}) d z d x d y=\int_{\Omega_{x y}} \mathbf{V}^{* T}(x, y) \mathbf{J G}(x, y) d x d y,
$$

with

$$
\mathbf{J}=\int_{\Omega_{z}} \hat{\mathbf{J}}(z) d z
$$

Integrating in the mesh $\Omega_{x y}=\cup_{e=1}^{E} \Omega_{x y}^{e}$,

$$
\int_{\Omega_{x y}} \mathbf{V}^{* T}(x, y) \mathbf{J G}(x, y) d x d y=\sum_{e=1}^{\mathrm{E}} \int_{\Omega_{x y}^{e}} \mathbf{V}^{e * T}(x, y) \mathbf{J} \mathbf{G}^{e}(x, y) d x d y,
$$

where $\mathbf{V}^{e}(x, y)$ and $\mathbf{G}^{e}(x, y)$ are approximated respectively from

$$
\mathbf{V}^{e}(x, y)=\mathbf{N}(x, y) \mathbf{U}^{e},
$$

and

$$
\mathbf{G}^{e}(x, y)=\mathbf{N}(x, y) \mathbf{R}^{e},
$$

with $\mathbf{R}^{e}$ containing the nodal values of $\mathbf{G}(x, y)$ and $\mathbf{N}(x, y)=\left[\mathbf{N}_{1}(x, y) \mathbf{N}_{2}(x, y) \mathbf{N}_{3}(x, y)\right]$, and

$$
\mathbf{N}_{i}=\left(\begin{array}{ccc}
N_{i}^{e}(x, y) & 0 & 0 \\
0 & N_{i}^{e}(x, y) & 0 \\
0 & 0 & N_{i}^{e}(x, y)
\end{array}\right)
$$

Thus, it results

$$
\begin{aligned}
& \sum_{e=1}^{E} \int_{\Omega_{x y}^{e}} \mathbf{V}^{e * T}(x, y) \mathbf{J} \mathbf{G}^{e}(x, y) d x d y=\sum_{e=1}^{E} \mathbf{U}^{e * T}\left\{\int_{\Omega_{x y}^{e}} \mathbf{N}^{T} \mathbf{J N} d x d y\right\} \mathbf{R}^{e} \\
& \quad=\sum_{e=1}^{E} \mathbf{U}^{e * T} \mathbf{A}_{x y}^{e} \mathbf{R}^{e}=\sum_{e=1}^{E} \mathbf{U}^{e * T} \mathbf{B}_{x y}^{e}=\mathbf{U}^{* T} \mathbf{B}_{x y}
\end{aligned}
$$

from which, the principle of virtual works reads

$$
\mathbf{U}^{* T} \mathbf{K}_{x y} \mathbf{U}=\mathbf{U}^{* T} \mathbf{B}_{x y} .
$$


Remark 1 In general the displacement decomposition within the PGD rationale involves more than a single mode, however, within the updating process, when calculating the $n$ mode, the $n-1$ already computed move to the right hand member, acting as generalized body force.

Remark 2 Thus, the in-plane functions determining the kinematics can be obtained from a standard plate theory software by using the elementary rigidity and forces given respectively by $\mathbf{K}_{x y}^{e}$ and $\mathbf{B}_{x y}^{e}$ considered in expression (26) and (38).

Remark 3 If traction $\mathbf{T} \neq \mathbf{0}$ the same procedure can be applied to treat the corresponding terms.

\section{Calculation of the out-of plane functions}

The expression of solution obtained in the previous section is given by

$$
\mathbf{u}(x, y, z)=\left(\begin{array}{c}
u(x, y, z) \\
v(x, y, z) \\
w(x, y, z)
\end{array}\right)=\left(\begin{array}{c}
\theta^{x}(x, y) f^{x}(z) \\
\theta^{y}(x, y) f^{y}(z) \\
w(x, y) f^{z}(z)
\end{array}\right)=\mathbf{V}(x, y) \circ \mathbf{W}(z),
$$

where

$$
\mathbf{V}(x, y)=\left(\begin{array}{c}
\theta^{x}(x, y) \\
\theta^{y}(x, y) \\
w(x, y)
\end{array}\right)
$$

and

$$
\mathbf{W}(z)=\left(\begin{array}{l}
f^{x}(z) \\
f^{y}(z) \\
f^{z}(z)
\end{array}\right)
$$

Now, we proceed to updated the out-of-plane functions involved in $\mathbf{W}(z)$ from the just calculated in-plane functions $\mathbf{V}(x, y)$ by considering again the principle of virtual work

$$
\int_{\Omega_{x y} \times \Omega_{z}} \boldsymbol{\varepsilon}\left(\mathbf{u}^{*}\right) \cdot\left(\mathbf{C} \cdot \boldsymbol{\varepsilon}(\mathbf{u}) d \mathbf{x}=\int_{\Omega_{x y} \times \Omega_{z}} \mathbf{u}^{*} \cdot \mathbf{f} d \mathbf{x}\right.
$$

where now in Eq. (40) the in-plane functions are assumed known and we look for the ones involved in the out-of-plane contribution $\mathbf{W}(z)$. Thus, the previous integral form can be integrated on $\Omega_{x y}$, and then Eq. (43) reduced to a one dimensional problem in $\Omega_{z}$ involving as unknown functions $f^{x}(z), f^{y}(z)$ and $f^{z}(z)$.

The same rationale that was previously addressed when performing the in-plane calculations is considered again but now with the test functions given by

$$
\boldsymbol{\varepsilon}^{*}=\boldsymbol{\Theta}^{1}(x, y) \circ \mathbf{F}^{1 *}(z)+\boldsymbol{\Theta}^{2}(x, y) \circ \mathbf{F}^{2 *}(z),
$$

and

$$
\mathbf{u}^{*}(x, y, z)=\mathbf{V}(x, y) \circ \mathbf{W}^{*}(z) .
$$

Now, from the updated out-of-plane functions in $\mathbf{W}(z)$, the in-plane functions in $\mathbf{V}(x, y)$ are again updated and the procedure repeats until reaching the convergence (fixed point). The procedure for computing the out-of-plane components in this minimally-intrusive way is detailed in Appendix A. 


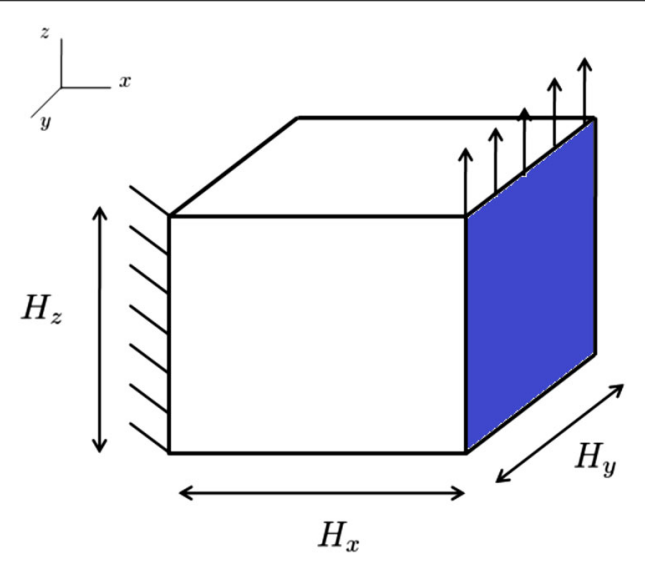

Fig. 1 The problem taken into consideration

Table 1 Model parameters

\begin{tabular}{ll}
\hline$H_{x}:$ Length in the $x$ direction $(\mathrm{mm})$ & 250 \\
$H_{y}$ : Length in the $y$ direction $(\mathrm{mm})$ & 250 \\
$H_{z}$ : Length in the $z$ direction $(\mathrm{mm})$ & 250 \\
$E$ : Young modulus $\left(\mathrm{N} / \mathrm{m}^{2}\right)$ & $2 \cdot 10^{11}$ \\
$\nu$ : Poisson coefficient & 0.25 \\
\hline
\end{tabular}

\section{Numerical results}

The problem taken into consideration is depicted in Fig. 1. The geometrical and mechanical properties of the plate domain $\Omega=\left[0, H_{x}\right] \times\left[0, H_{y}\right] \times\left[0, H_{z}\right]$ are defined in Table 1 On the right boundary face of the domain (the blue zone in Fig. 1) a vertical traction is enforced, $\mathbf{T}=\left(0,0,8 \cdot 10^{9}\right) \mathrm{N} / \mathrm{m}^{2}$ and on the opposite face homogeneous Dirichlet boundary conditions are imposed. No volumetric body forces are considered. As in the considered domain the thickness (out-of-plane) dimension is not much lower than the other ones (in-plane dimensions), the linear variation of the displacement field along the thickness described by (2) is not more true as we can notice in Fig. 2 that compares the plate solution from the fully $3 \mathrm{D}$ solution assumed as reference. However using the just proposed minimally-intrusive fully $3 \mathrm{D}$ separated plate formulation we can notice how the solution is improved. Figure 3 shows the error of the solution respect to the 3D FEM solution, computed as

$$
\xi(\mathbf{u})=\frac{\left(\int_{\Omega}\left(\mathbf{u}-\mathbf{u}_{F E M}\right)^{2} d \mathbf{x}\right)^{\frac{1}{2}}}{\left(\int_{\Omega}\left(\mathbf{u}_{F E M}\right)^{2} d \mathbf{x}\right)^{\frac{1}{2}}},
$$

as a function of the number of modes. The error of the plate theory solution results $\xi\left(\mathbf{u}_{\text {plate }}\right)=0.0633$.

We consider now the same problem as in the previous example but this time we suppose that there is an hole in the domain. As in the previous example, in Fig. 4 it is depicted the solution computed using the different techniques. Moreover in Figs. 5, 6 and 7 different perspectives of the out-of-plane stress tensor components are depicted. Let's note how the proposed method is able to take into consideration the $\sigma_{z z}$ components, which is ignored 


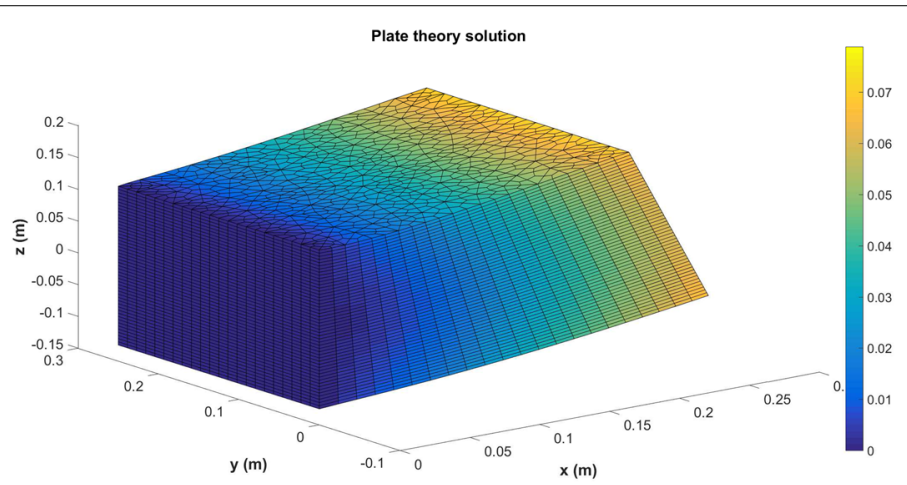

$\mathbf{a}$

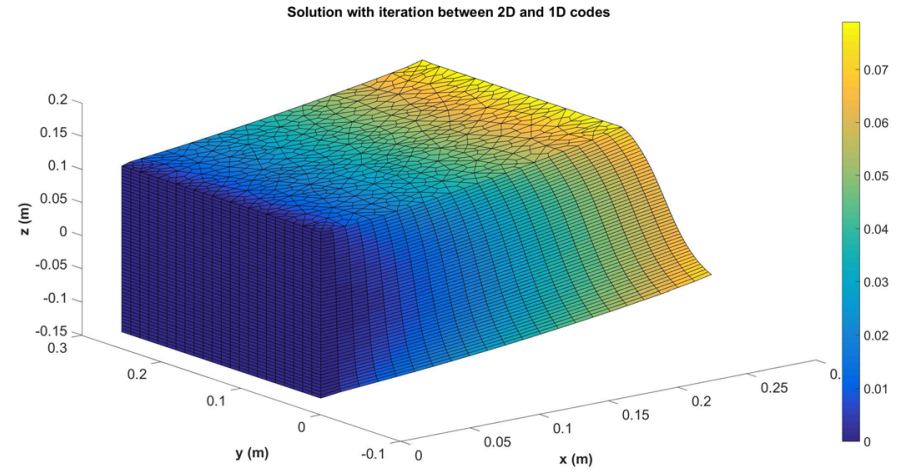

b

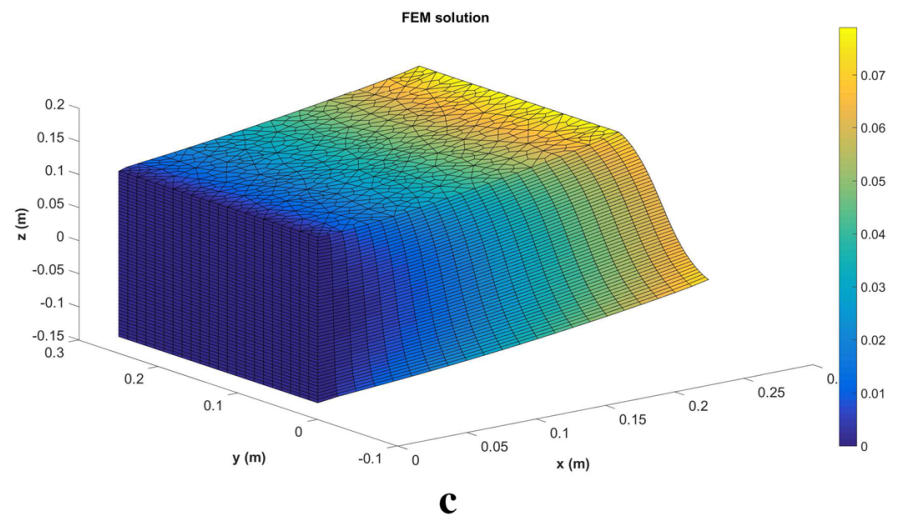

Fig. 2 Displacement field using: plate theory (a), minimally-intrusive fully 3D separated plate formulation (b), 3D FEM (c)

in plate theory, and to obtain the parabolic evolution around the thickness for the $\sigma_{x z}$ and $\sigma_{y z}$ typical of a 3D solution (Fig. 7).

In Figs. 8, 9 and 10 the same quantities are computed using a 3D finite element method, proving the good accuracy of the proposed method.

Again, in Fig. 11 shows the error of the solution respect to the 3D FEM solution as a function of the number of modes. The error of the plate theory solution being $\xi\left(\mathbf{u}_{\text {plate }}\right)=$ 0.0638 . 


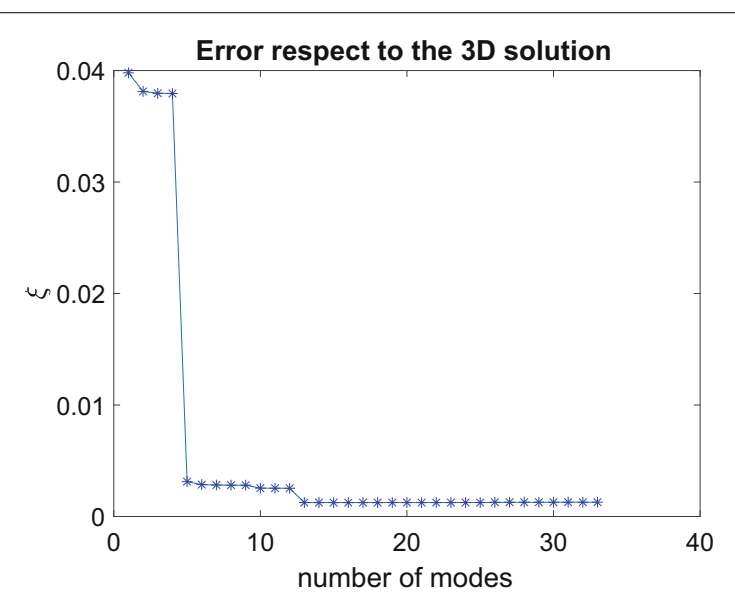

Fig. 3 Error of the enriched solution respect to the 3D solution for different number of modes

\section{Extension of the method to elasto-plastic dynamics}

In this section we extend the method to dynamics problem in which plastic behavior can occur. With $\mathbf{g}(\mathbf{x}, t)$ the body forces, the displacement field evolution $\mathbf{u}(\mathbf{x}, t)$ in the domain $\Omega$ and time interval $t \in I=(0, T]$ is described by the linear momentum balance equation

$$
\rho \ddot{\mathbf{u}}(\mathbf{x}, t)=\nabla \cdot \sigma+\mathbf{g},
$$

where $\rho$ is the density $\left(\mathrm{kg} / \mathrm{m}^{3}\right)$.

The boundary $\partial \Omega$ is decomposed according to $\partial \Omega=\Gamma_{D} \cup \Gamma_{N}$ where displacement and tractions $\mathbf{T}(t)$ are prescribed.

The behavior relating the Cauchy's stress $\sigma$ and the elastic strain $\varepsilon^{e}$ reads [5]

$$
\sigma=\mathbf{C} \varepsilon^{e}=\mathbf{C}\left(\varepsilon-\varepsilon^{p}\right),
$$

where $\mathbf{C}$ is the Hooke tensor, $\boldsymbol{\varepsilon}$ is total strain and $\boldsymbol{\varepsilon}^{p}$ is the plastic strain.

The problem weak form associated with the strong form (47) results in looking for the displacement field $\mathbf{u}$ verifying the initial and Dirichlet boundary conditions, and fulfilling

$$
\rho \int_{\Omega} \mathbf{u}^{*} \cdot \ddot{\mathbf{u}} d \mathbf{x}+\int_{\Omega} \boldsymbol{\varepsilon}\left(\mathbf{u}^{*}\right) \cdot\left(\mathbf{C}\left(\boldsymbol{\varepsilon}(\mathbf{u})-\boldsymbol{\varepsilon}^{p}(\mathbf{u})\right)\right) d \mathbf{x}=\int_{\Omega} \mathbf{u}^{*} \cdot \mathbf{g} d \mathbf{x}+\int_{\Gamma_{N}} \mathbf{u}^{*} \cdot \mathbf{T}(t) d \mathbf{x}
$$

for any test function $\mathbf{u}^{*}$ in an appropriate functional space.

We consider at time $t_{j+1}$ the standard explicit time integration [6] (widely considered in commercial codes) given by

$$
\begin{aligned}
& \rho \int_{\Omega} \mathbf{u}^{*} \cdot \frac{\mathbf{u}^{j+1}-2 \mathbf{u}^{j}+\mathbf{u}^{j-1}}{\Delta t^{2}} d \mathbf{x}+\int_{\Omega} \boldsymbol{\varepsilon}\left(\mathbf{u}^{*}\right) \cdot\left(\mathbf{C}\left(\boldsymbol{\varepsilon}\left(\mathbf{u}^{j}\right)-\boldsymbol{\varepsilon}^{p}\left(\mathbf{u}^{j}\right)\right)\right) d \mathbf{x} \\
& \quad=\int_{\Omega} \mathbf{u}^{*} \cdot \mathbf{g}^{j} d \mathbf{x}+\int_{\Gamma_{N}} \mathbf{u}^{*} \cdot \mathbf{T}^{j} d \mathbf{x},
\end{aligned}
$$

or, by putting all the explicit terms at the right hand side, 


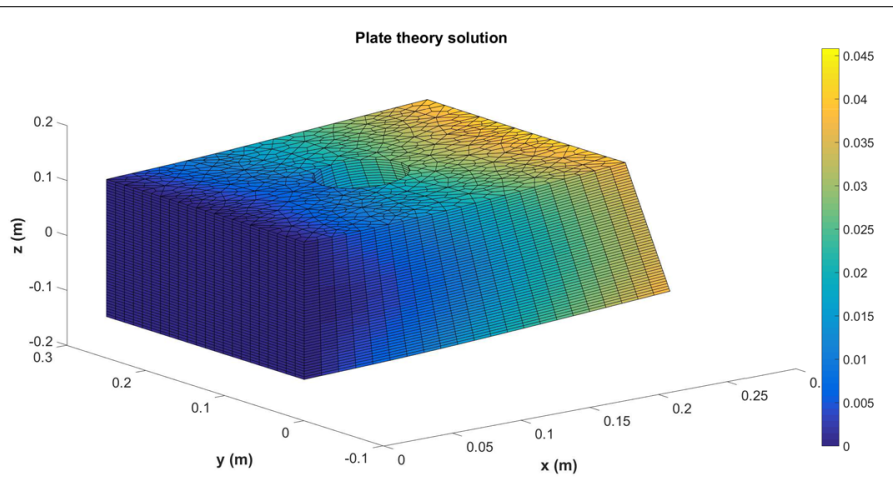

a

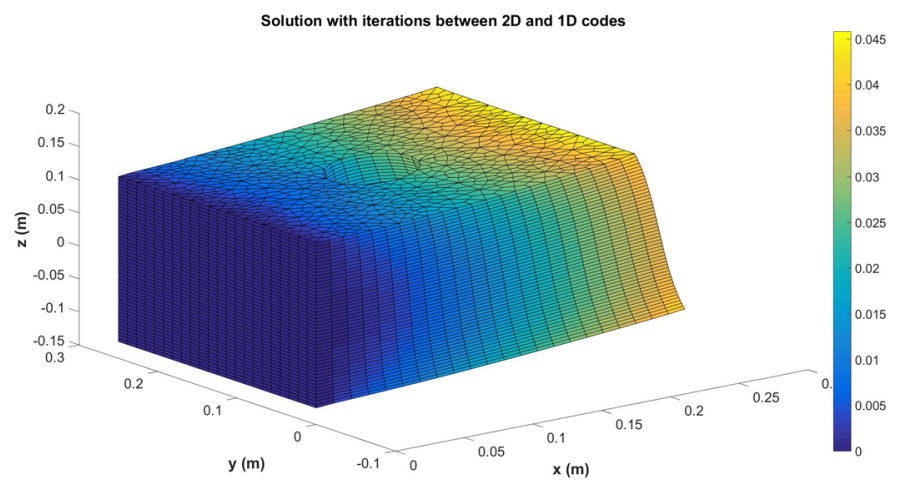

b

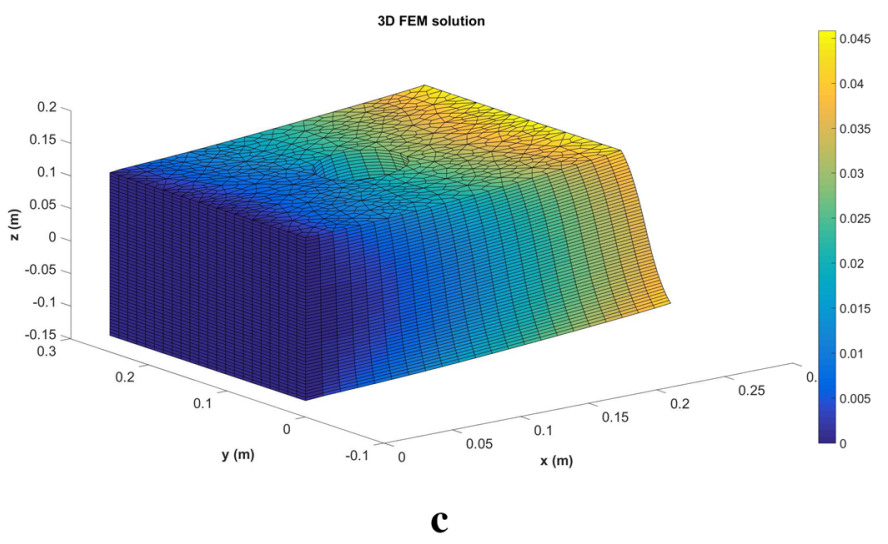

Fig. 4 Displacement field using: plate theory (a), minimally-intrusive fully 3D separated plate formulation (b), $3 \mathrm{DFEM}(\mathbf{c})$

$$
\begin{aligned}
& \rho \int_{\Omega} \mathbf{u}^{*} \cdot \mathbf{u}^{j+1} d \mathbf{x}=\rho \int_{\Omega} \mathbf{u}^{*} \cdot\left(2 \mathbf{u}^{j}-\mathbf{u}^{j-1}\right) d \mathbf{x} \\
& \quad-\Delta t^{2}\left(\int_{\Omega} \boldsymbol{\varepsilon}\left(\mathbf{u}^{*}\right) \cdot\left(\mathbf{C}\left(\boldsymbol{\varepsilon}\left(\mathbf{u}^{j}\right)-\boldsymbol{\varepsilon}^{p}\left(\mathbf{u}^{j}\right)\right)\right) d \mathbf{x}+\int_{\Omega} \mathbf{u}^{*} \cdot \mathbf{g}^{j} d \mathbf{x}+\int_{\Gamma_{N}} \mathbf{u}^{*} \cdot \mathbf{T}^{j} d \mathbf{x}\right) .
\end{aligned}
$$



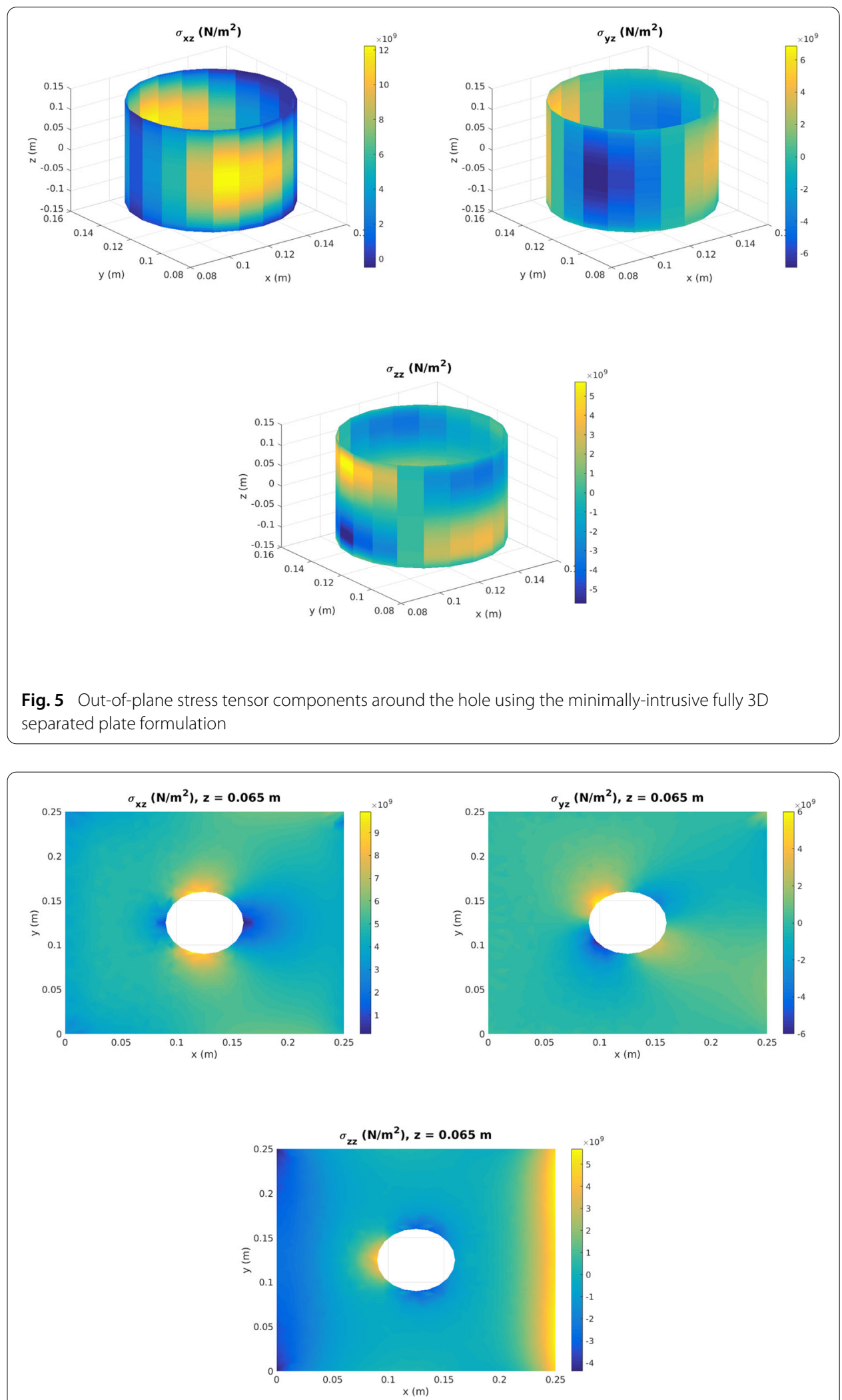

Fig. 6 Out-of-plane stress tensor components in the $z=65 \mathrm{~mm}$ plane using the minimally-intrusive fully $3 \mathrm{D}$ separated plate formulation 


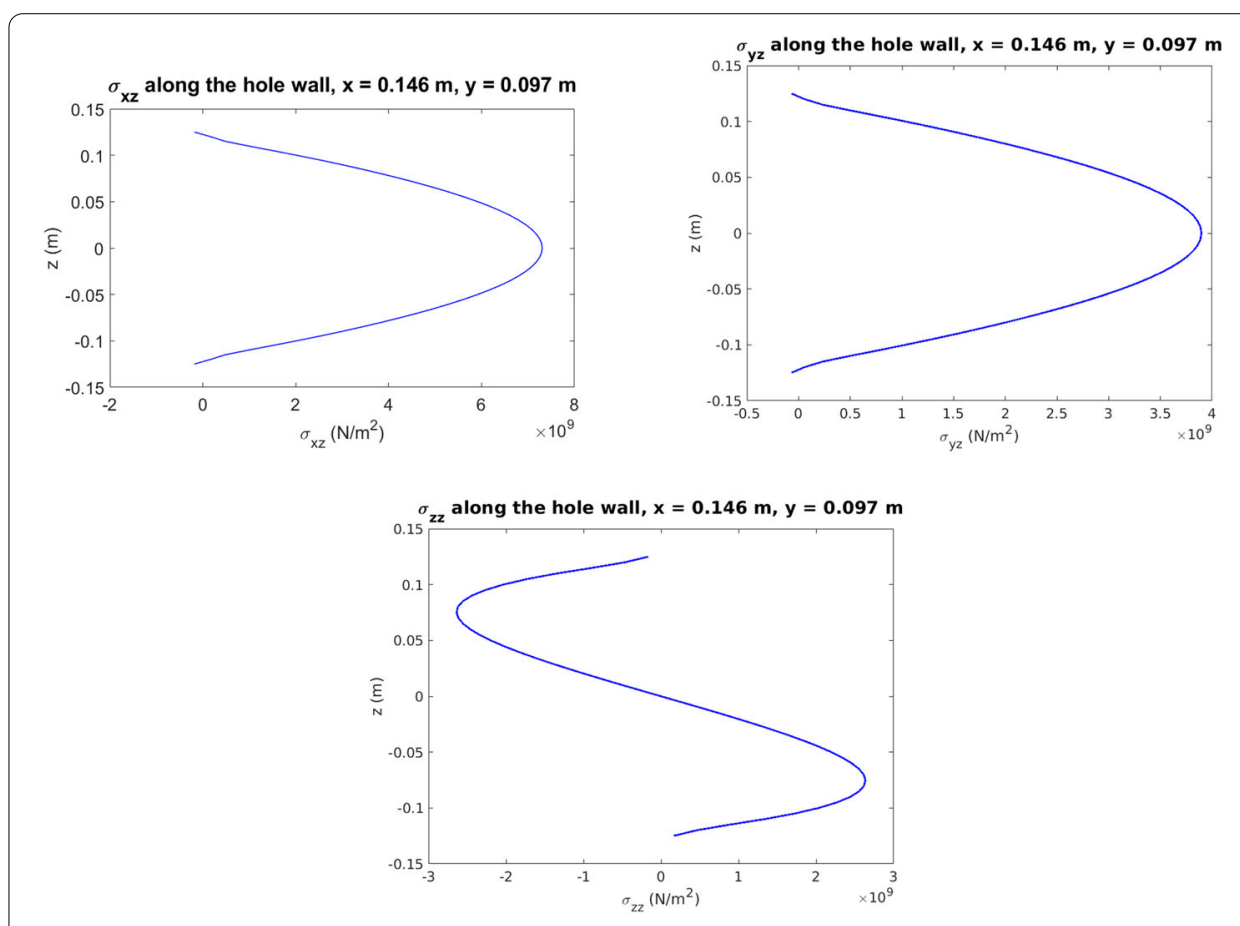

Fig. 7 Out-of-plane stress tensor components around the hole for $x=146 \mathrm{~mm}$ and $y=97 \mathrm{~mm}$ using the minimally-intrusive fully 3D separated plate formulation

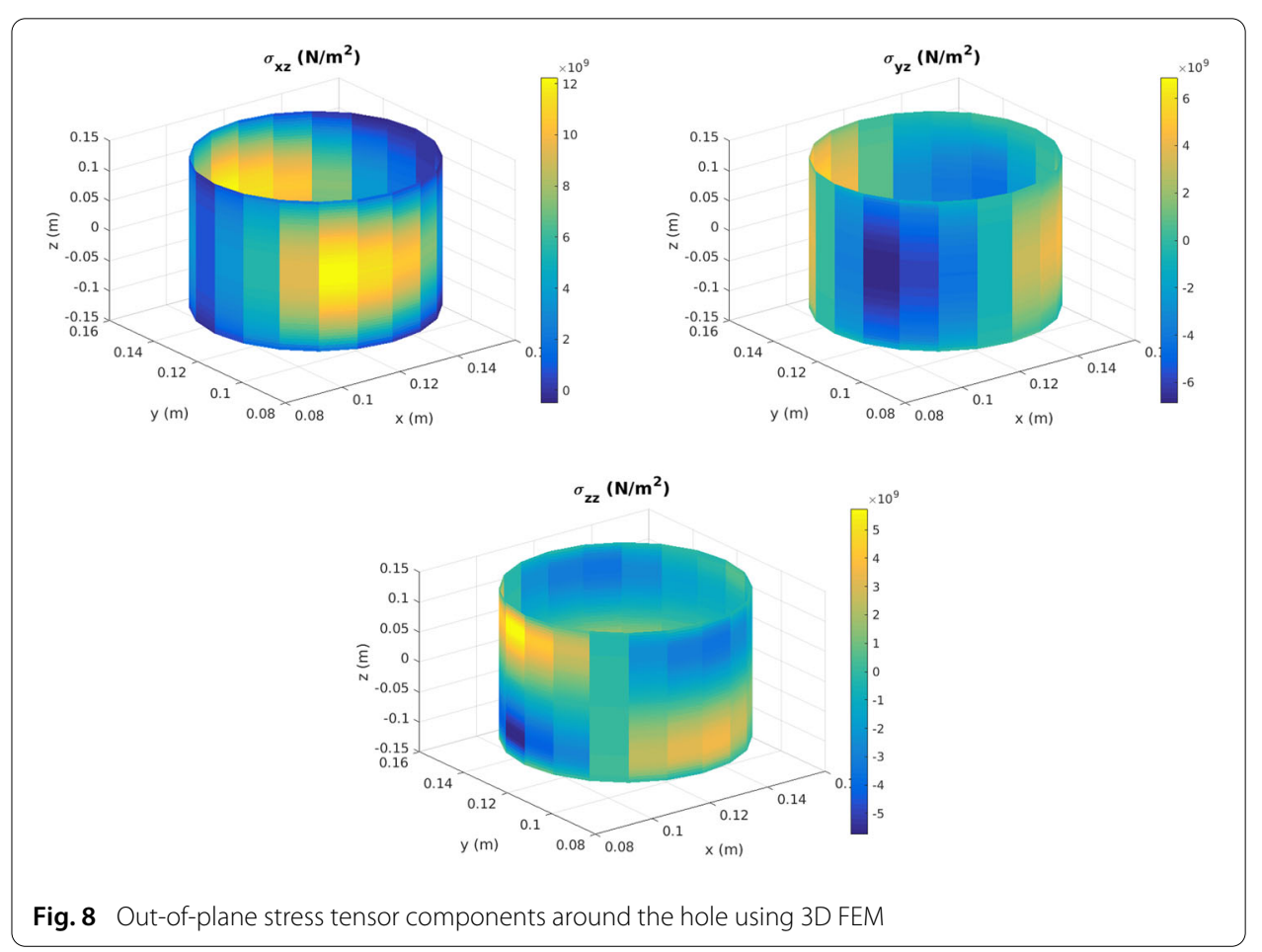



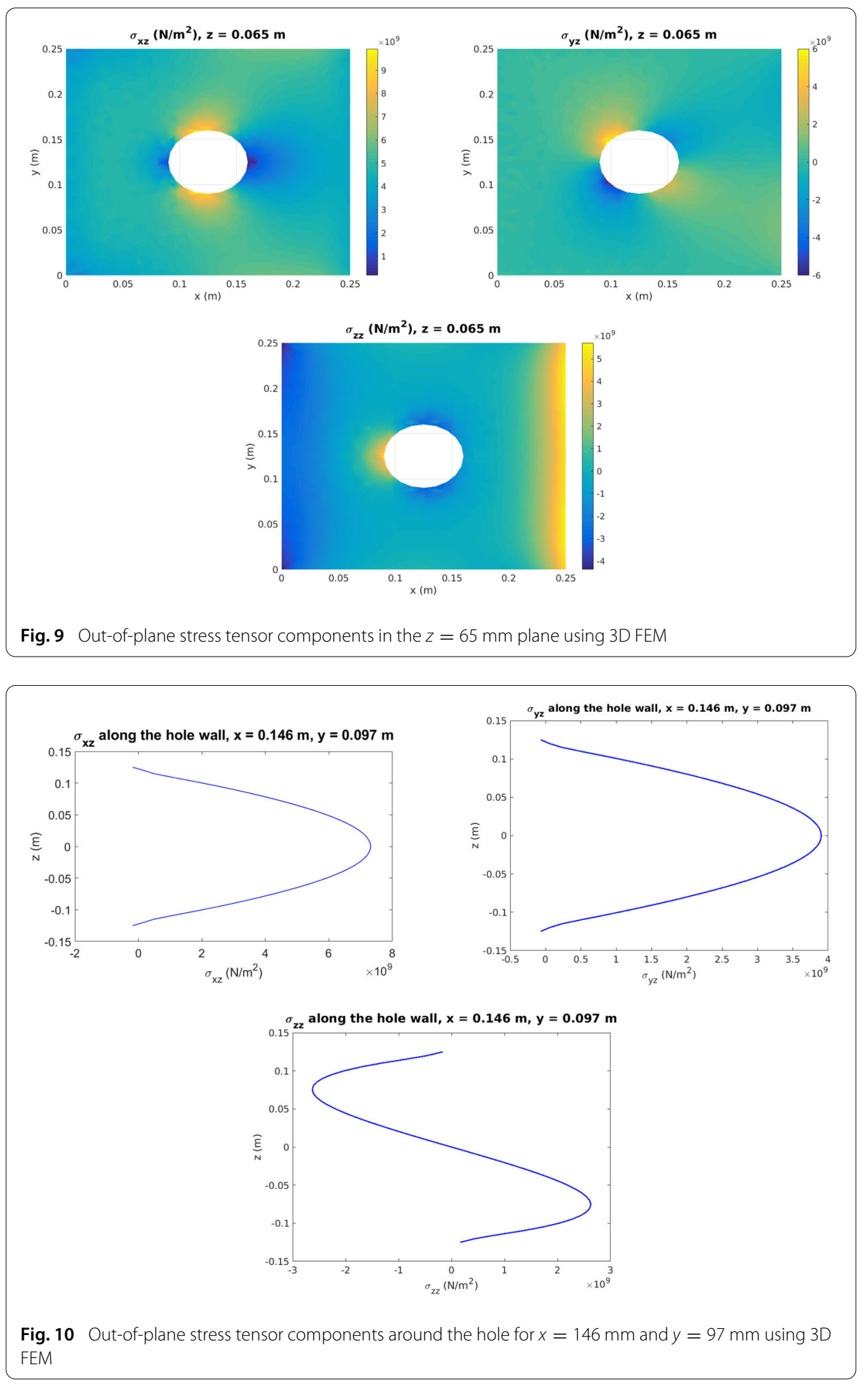


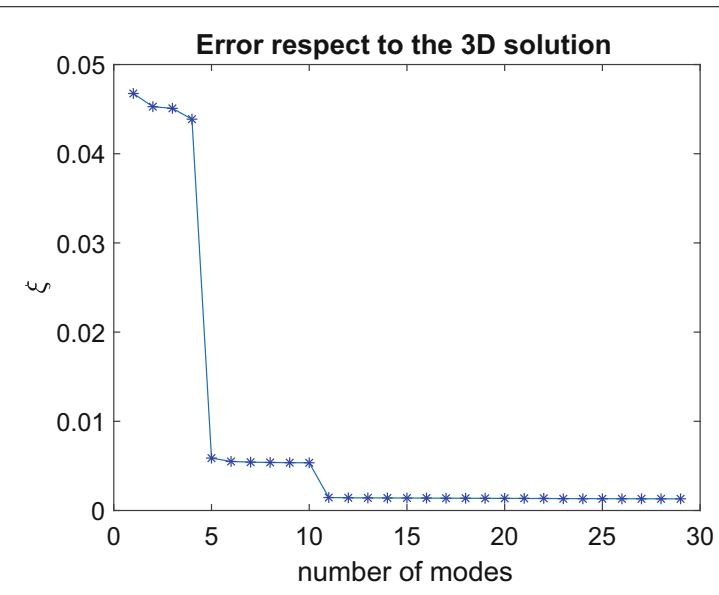

Fig. 11 Error of the enriched solution respect to the 3D solution for different number of modes

Recalling (28) we can write

$$
\mathbf{u}^{j+1}(x, y, z)=\mathbf{V}^{j+1} \circ \mathbf{W}^{j+1} .
$$

Supposing the out-of-plane functions known, the left hand side term in (51) can be expressed as

$$
\mathbf{u}^{* T} \mathbf{u}^{j+1}(\mathbf{x})=\mathbf{V}^{j+1, * T}(x, y) \hat{\mathbf{J}}^{j+1}(z) \mathbf{V}^{j+1}(x, y),
$$

where matrix $\hat{\mathbf{J}}^{j+1}$ reads

$$
\hat{\mathbf{J}}_{k l}^{j+1}(z)=\mathbf{I}_{k l} \mathbf{W}_{k}^{j+1}(z) \mathbf{W}_{l}^{j+1}(z),
$$

with I the identity matrix.

Now, the integral results

$$
\rho \int_{\Omega} \mathbf{u}^{*} \cdot \mathbf{u}^{j+1} d z d x d y=\int_{\Omega_{x y}} \mathbf{V}^{j+1, * T}(x, y) \mathbf{J}^{j+1} \mathbf{V}^{j+1}(x, y) d x d y,
$$

with

$$
\mathbf{J}^{j+1}=\int_{\Omega_{z}} \hat{\mathbf{J}}^{j+1}(z) d z
$$

Integrating in the mesh $\Omega_{x y}=\cup_{e=1}^{\mathrm{E}} \Omega_{x y}^{e}$,

$$
\int_{\Omega_{x y}} \mathbf{V}^{j+1, * T} \mathbf{J}^{j+1} \mathbf{V}^{j+1} d x d y=\sum_{e=1}^{\mathrm{E}} \int_{\Omega_{x y}^{e}} \mathbf{V}^{j+1, e * T} \mathbf{J}^{j+1} \mathbf{V}^{j+1, e} d x d y,
$$

where $\mathbf{V}^{j+1, e}(x, y)$ is approximated from

$$
\mathbf{V}^{j+1, e}(x, y)=\mathbf{N}(x, y) \mathbf{U}^{j+1, e},
$$

with $\mathbf{N}(x, y)=\left[\mathbf{N}_{1}(x, y) \mathbf{N}_{2}(x, y) \mathbf{N}_{3}(x, y)\right]$, and

$$
\mathbf{N}_{i}=\left(\begin{array}{ccc}
N_{i}^{e}(x, y) & 0 & 0 \\
0 & N_{i}^{e}(x, y) & 0 \\
0 & 0 & N_{i}^{e}(x, y)
\end{array}\right) .
$$




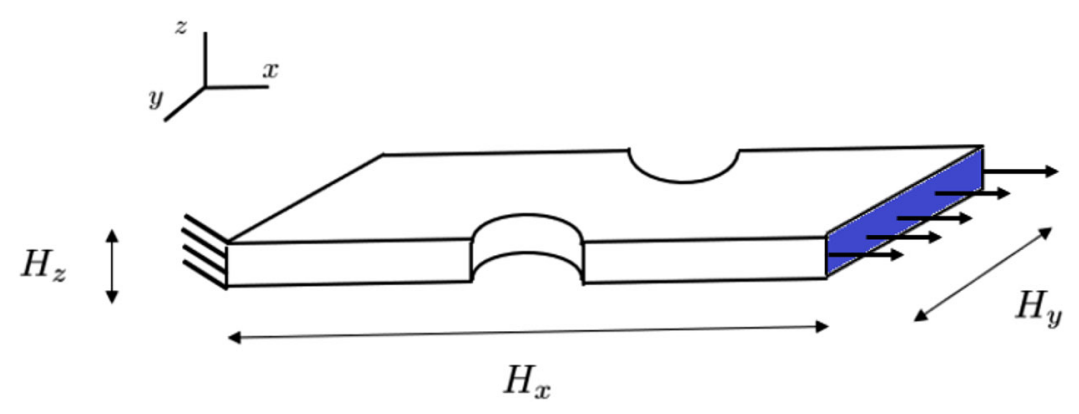

Fig. 12 The elasto-plastic dynamical problem taken into consideration

Table 2 Model parameters

\begin{tabular}{ll}
\hline$H_{x}$ : Length in the $x$ direction $(\mathrm{mm})$ & 250 \\
$H_{y}$ : Length in the $y$ direction $(\mathrm{mm})$ & 250 \\
$H_{z}$ : Length in the $z$ direction $(\mathrm{mm})$ & 20 \\
$E:$ Young modulus $\left(\mathrm{N} / \mathrm{m}^{2}\right)$ & $6.68 \cdot 10^{10}$ \\
$\nu:$ Poisson coefficient & 0.35 \\
$\rho:$ Density $\left(\mathrm{kg} / \mathrm{m}^{3}\right)$ & 2700
\end{tabular}

Thus, it results

$$
\begin{aligned}
& \sum_{e=1}^{E} \int_{\Omega_{x y}^{e}} \mathbf{V}^{j+1, e * T} \mathbf{J}^{j+1} \mathbf{V}^{j+1, e} d x d y=\sum_{e=1}^{E} \mathbf{U}^{j+1, e * T}\left\{\int_{\Omega_{x y}^{e}} \mathbf{N}^{T} \mathbf{J}^{j+1} \mathbf{N} d x d y\right\} \mathbf{U}^{j+1, e} \\
& =\sum_{e=1}^{E} \mathbf{U}^{j+1, e * T} \mathbf{M}_{x y}^{j+1, e} \mathbf{U}^{j+1, e}=\mathbf{U}^{j+1, * T} \mathbf{M}_{x y}^{j+1} \mathbf{U}^{j+1} .
\end{aligned}
$$

The different terms at the right hand side of (51) can be treated in a similar way, as already explained for the static case, so that at each time step $j$ the virtual work principle reads

$$
\mathbf{U}^{j+1, * T} \mathbf{M}_{x y}^{j+1} \mathbf{U}^{j+1}=\mathbf{U}^{j+1, * T} \mathbf{B}_{x y}^{j}
$$

Remark 4 As for the static case, the in-plane functions determining the kinematics can be obtained from a standard plate theory software by using the elementary mass and forces given respectively by $\mathbf{M}_{x y}^{j+1, e}$ and $\mathbf{B}_{x y}^{j, e}$.

Remark 5 Again the out-of-plane functions can be obtained in a similar manner as already explained in the static case.

For evaluating the performances of the method we consider the problem defined in Fig. 12. The geometrical and mechanical properties of the plate domain are defined in Table 2. On the right boundary face of the domain (the blue zone in Fig. 12) an horizontal traction is enforced, $\mathbf{T}=\left(2.7 \cdot 10^{8}, 0,0\right) \mathrm{N} / \mathrm{m}^{2}$ and on the opposite face homogeneous Dirichlet boundary conditions are imposed. No volumetric body forces are considered. For the sake of simplicity, we use the Von Mises criterion [7], assuming a Krupkowski isotropic hardening [8] given by the formula:

$$
\bar{\sigma}=K\left(\bar{\varepsilon}_{0}+\bar{\varepsilon}_{p}\right)^{p}
$$

where $\bar{\varepsilon}_{0}=0.008$ is the initial equivalent plastic strain, $\bar{\varepsilon}_{p}$ is the equivalent plastic strain, $K=0.4619 \mathrm{GPa}$ is a strength coefficient and $p=0.1$ is the strain hardening exponent 


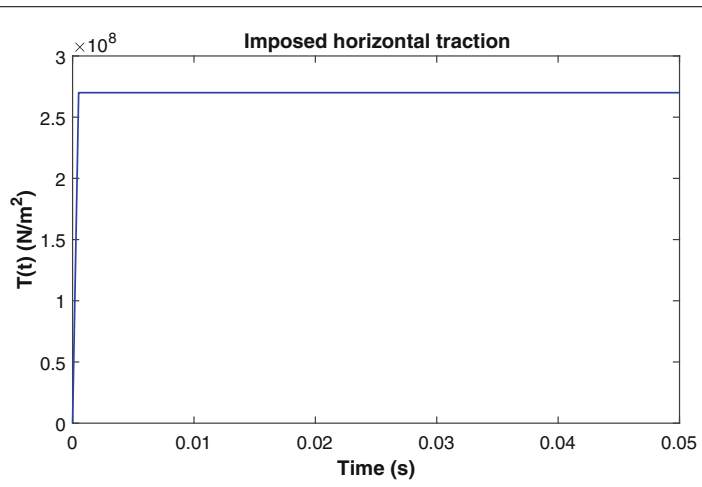

Fig. 13 Horizontal loading for the elasto-plastic dynamical problem

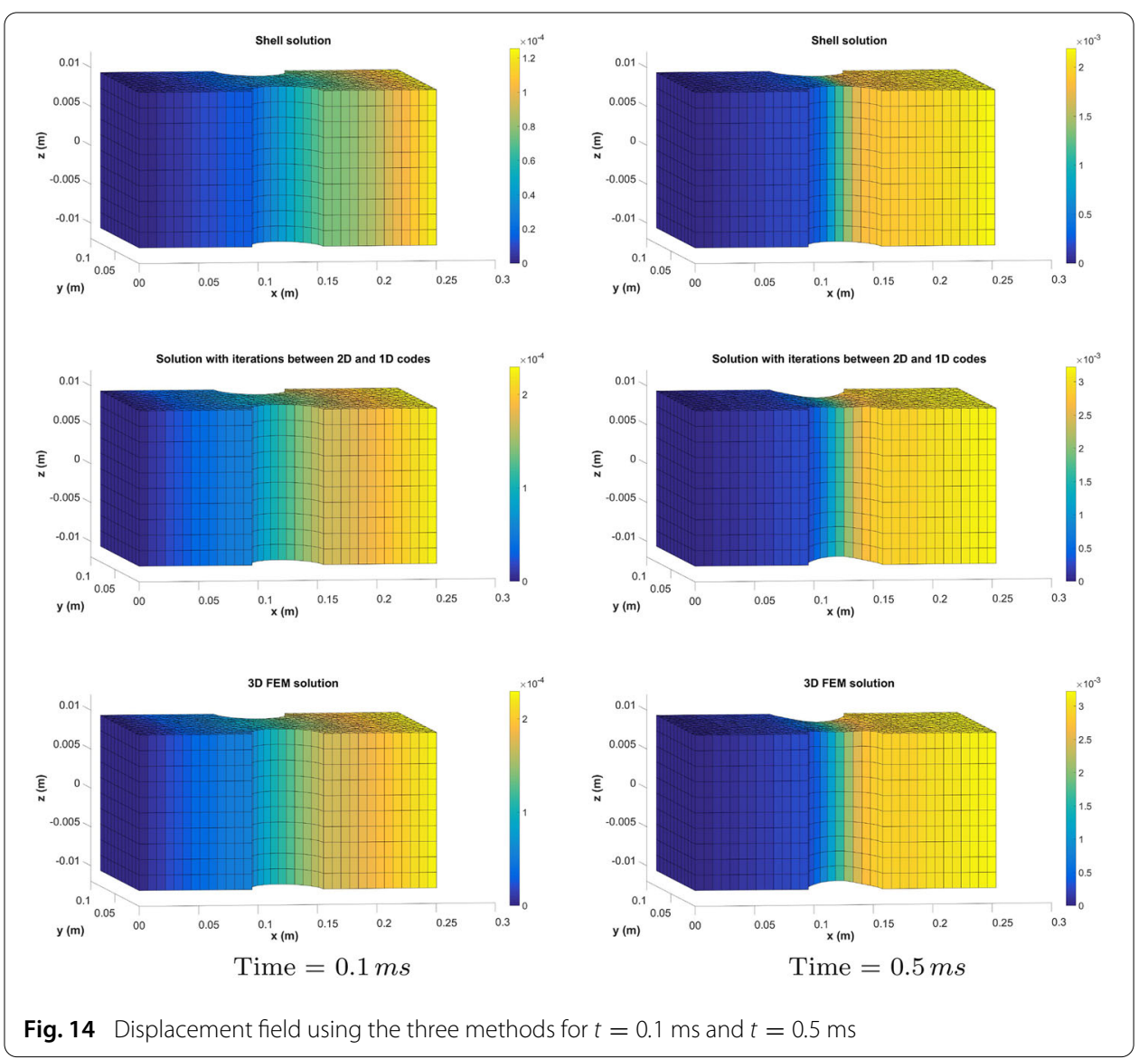

[9]. The problem is solved in the time interval $[0,50] \mathrm{ms}$ with a time step $\Delta t=10^{-4} \mathrm{~ms}$ which ensures the stability of the explicit method. In order to get the stationary solution the traction is applied gradually as depicted in Fig. 13 and a Rayleigh damping (proportional to the mass) is used. Figs. 14, 15 and 16 compares the solution obtained with the three methods at different times. For this problem the 2D solution is given by shell theory [1] as 

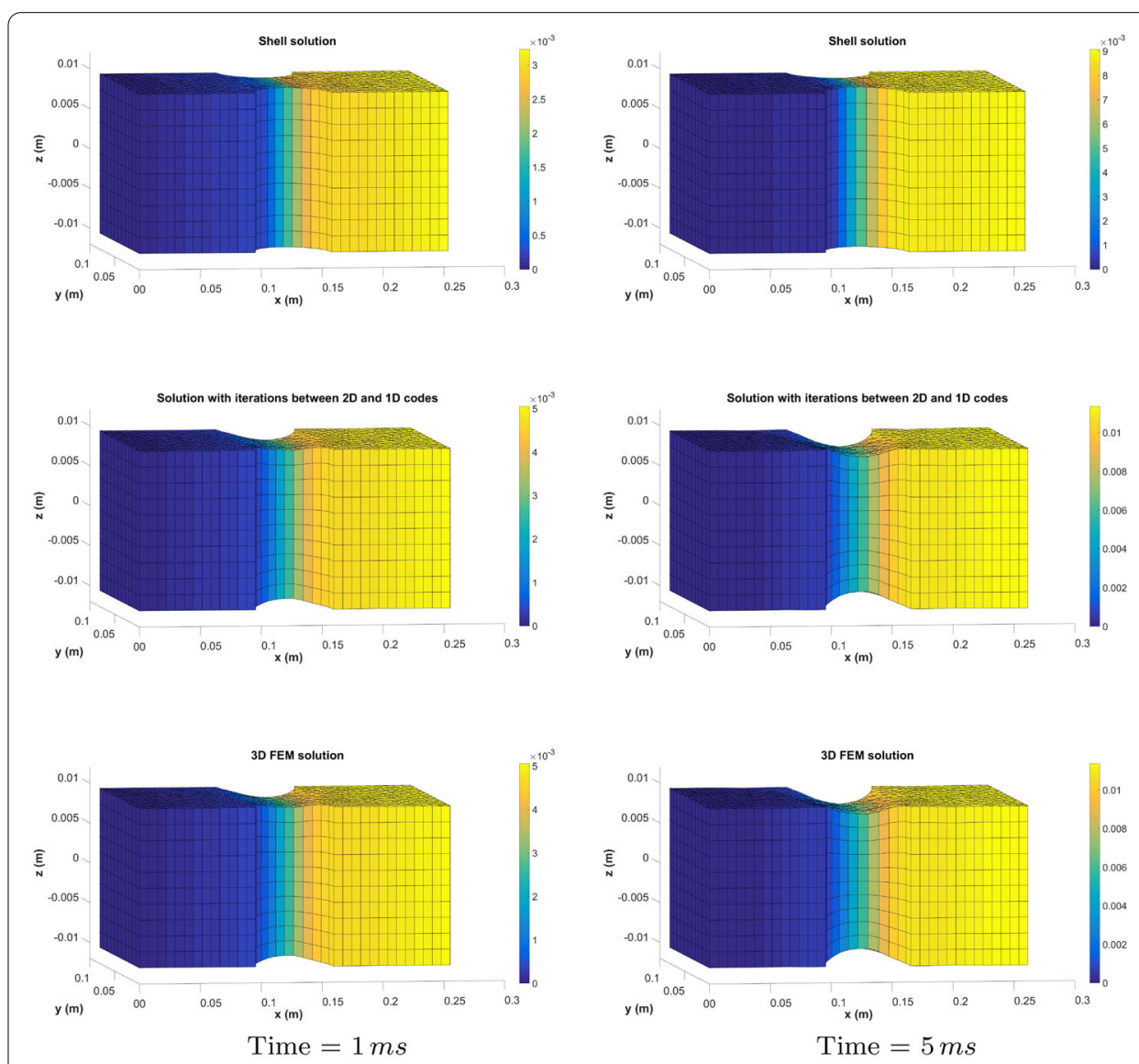

Fig. 15 Displacement field using the three methods for $t=1 \mathrm{~ms}$ and $t=5 \mathrm{~ms}$

$$
\left\{\begin{array}{l}
u(x, y, z)=u_{0}(x, y)-z \theta_{x}(x, y) \\
v(x, y, z)=v_{0}(x, y)-z \theta_{y}(x, y) \\
w(x, y, z)=w_{0}(x, y)
\end{array}\right.
$$

where $u_{0}, v_{0}$ and $w_{0}$ are the displacements of the points on the middle plane along $x, y$ and $z$ respectively and the rotations $\theta_{x}$ and $\theta_{y}$ coincide with the angles followed by the normal vectors contained in the planes $x z$ and $y z$ respectively in their motions.

Again the solution computed using the proposed method is able to get a 3D behavior (as the one of the 3D FEM solution) with the striction along the thickness in the zone with a smaller section, which is typical of a 3D plastic solution.

\section{Conclusions}

Here we proposed a minimally intrusive formulation of mechanical problems (linear, elasto-plastic, static and dynamic) defined in separable domains, enabling 3D solutions expressed as a finite sum decomposition involving the product of functions defined in the plane and in the thickness. The main contribution of the present work is that the calculation of functions defined in the plane, the most expensive computationally, can be ensured by a standard plate solver, whereas the solution of those defined in the thickness, defining 


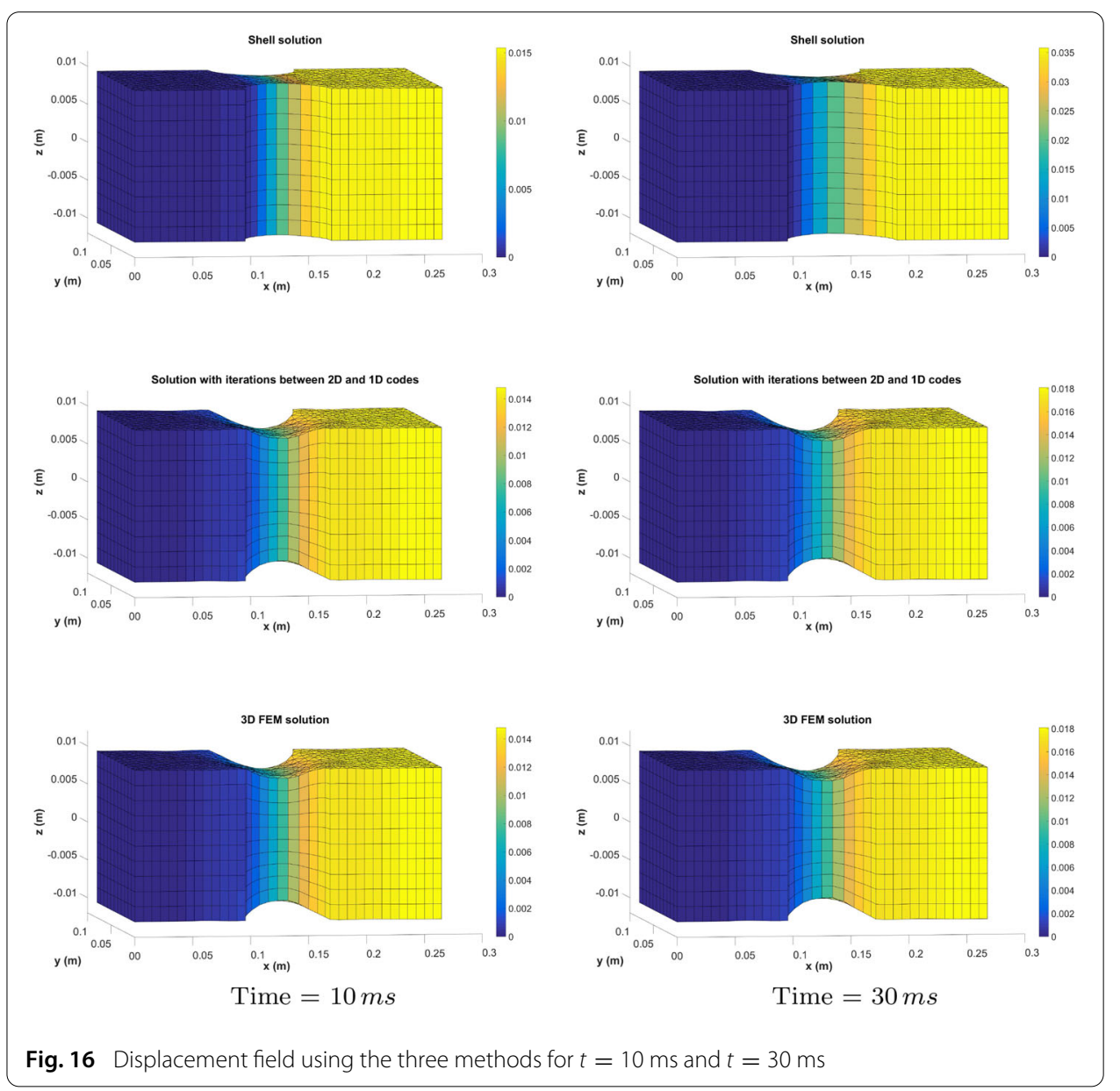

1D problems extremely simple and cheap, is externalized and ensured by a function called by the plate solver.

The different numerical examples prove the procedure efficiency that allows computing 3D solutions while keeping the computational cost the one characteristic of standard 2D plate and shell calculations.

\section{Authors' contributions}

$G Q, M Z, E H$, JLD and FC authors participated in the definition of techniques and algorithms. All authors read and approved the final manuscript.

\section{Author details}

'ESI Group, Parc Icade, Immeuble le Seville, 3 bis, Saarinen, CP 50229, 94528 Rungis Cedex, France, 2PIMM, ENSAM ParisTech \& ESI GROUP Chair on Advanced Modeling and Simulation of Manufacturing Processes, 151 Boulevard de I'Hopital, 75013 Paris, France.

\section{Acknowledgements}

Authors acknowledge the support of the ESI Group through its research chair at ENSAM ParisTech.

\section{Funding}

This project has received funding from the European Union's Horizon 2020 research and innovation programme under the Marie Skłodowska-Curie Grant Agreement No. 675919.

\section{Availability of data and materials}

Interested reader can contact authors.

\section{Competing interests}

The authors declare that they have no competing interests. 


\section{Appendix A: Calculation of the out-of plane functions in a minimally-intrusive} manner

We write the virtual work principle

$$
\begin{aligned}
\boldsymbol{\varepsilon}^{* T} \boldsymbol{\sigma} & =\boldsymbol{\varepsilon}^{* T} \mathbf{C} \boldsymbol{\varepsilon} \\
& =\left\{\boldsymbol{\Theta}^{1}(x, y) \circ \mathbf{F}^{1 *}(z)+\boldsymbol{\Theta}^{2}(x, y) \circ \mathbf{F}^{2 *}(z)\right\}^{T}\left\{\mathbf{C}_{x y}(x, y) \circ \mathbf{C}_{z}(z)\right\} \\
& \left\{\boldsymbol{\Theta}^{1}(x, y) \circ \mathbf{F}^{1}(z)+\boldsymbol{\Theta}^{2}(x, y) \circ \mathbf{F}^{2}(z)\right\} \\
& =\mathbf{F}^{1 * T}(x, y)\left\{\hat{\mathbf{C}}_{x y}^{11}(x, y) \circ \mathbf{C}_{z}(z)\right\} \mathbf{F}^{1}(x, y)+\mathbf{F}^{1 * T}(x, y)\left\{\hat{\mathbf{C}}_{x y}^{12}(x, y) \circ \mathbf{C}_{z}(z)\right\} \\
& \mathbf{F}^{2}(x, y)+\mathbf{F}^{2 * T}(x, y)\left\{\hat{\mathbf{C}}_{x y}^{21}(x, y) \circ \mathbf{C}_{z}(z)\right\} \mathbf{F}^{1}(x, y)+\mathbf{F}^{2 * T}(x, y) \\
& \left\{\hat{\mathbf{C}}_{x y}^{22}(x, y) \circ \mathbf{C}_{z}(z)\right\} \mathbf{F}^{2}(x, y) .
\end{aligned}
$$

In the previous expression matrices $\hat{\mathbf{C}}_{x y}^{i j}(x, y)$ results

$$
\hat{\mathbf{C}}_{x y_{k l}}^{i j}(x, y)=\mathbf{C}_{x y_{k l}}(x, y) \boldsymbol{\Theta}_{k}^{i}(x, y) \boldsymbol{\Theta}_{l}^{j}(x, y), \quad i, j \in[1,2] \& \quad k, l \in[1, \cdots, 6] .
$$

Now, the virtual work integral reads

$$
\begin{aligned}
& \int_{\Omega_{x y} \times \Omega_{z}} \sum_{i=1}^{2} \sum_{j=1}^{2} \mathbf{F}^{i * T}(z)\left\{\hat{\mathbf{C}}_{x y}^{i j}(x, y) \circ \mathbf{C}_{z}(z)\right\} \mathbf{F}^{j}(z) d z d x d y \\
& =\int_{\Omega_{z}} \sum_{i=1}^{2} \sum_{j=1}^{2} \mathbf{F}^{i * T}(z) \mathbf{P}^{i j}(z) \mathbf{F}^{j}(z) d z,
\end{aligned}
$$

where

$$
\mathbf{P}^{i j}(z)=\mathbf{C}_{z}(z) \circ \int_{\Omega_{x y}} \hat{\mathbf{C}}_{x y}^{i j}(x, y) d x d y .
$$

Now, if we assume for instance an approximation based on piecewise linear interpolations on the 1D finite element mesh of $\Omega_{z}=\cup_{q=1}^{Q} \Omega_{z}^{q}$, with the shape functions defined by $N_{i}^{q}(z), i=1,2 ; q=1, \ldots, Q ;$ it results

$$
\left\{\begin{array}{l}
f^{x, q}(z)=N_{1}^{q}(z) f_{1}^{x, q}+N_{2}^{q}(z) f_{2}^{x, q} \\
f^{y, q}(z)=N_{1}^{q}(z) f_{1}^{y, q}+N_{2}^{q}(z) f_{2}^{y, q} \\
f^{z, q}(z)=N_{1}^{q}(z) f_{1}^{z, q}+N_{2}^{q}(z) f_{2}^{z, q}
\end{array}\right.
$$

Using that approximation we can express vectors $\mathbf{F}^{i}(z)$ in each element $\Omega_{z}^{q}$ from

$$
\mathbf{L}^{q T}=\left(f_{1}^{x, q}, f_{1}^{y, q}, f_{1}^{z, q}, f_{2}^{x, q}, f_{2}^{y, q}, f_{2}^{z, q}\right),
$$

and

$$
\mathbf{F}^{i}\left(z \in \Omega_{z}^{q}\right)=\mathbf{T}^{i, q}(z) \mathbf{L}^{q},
$$

where $\mathbf{T}^{i, q}(z)$ contains the shape functions and theirs derivatives, according to the expressions involved in the components of $\mathbf{F}^{i}(z), i=1,2$. 
Thus, integral (66) reads

$$
\begin{gathered}
\sum_{q=1}^{Q} \mathbf{L}^{q * T}\left\{\int_{\Omega_{z}^{q}} \sum_{i=1}^{2} \sum_{j=1}^{2} \mathbf{T}^{i, q T}(z) \mathbf{P}^{i j}(z) \mathbf{T}^{j, q}(z) d z\right\} \mathbf{L}^{q} \\
=\sum_{q=1}^{Q} \mathbf{L}^{q * T} \mathbf{K}_{z}^{q} \mathbf{L}^{q}=\mathbf{L}^{* T} \mathbf{K}_{z} \mathbf{L} .
\end{gathered}
$$

The virtual work (27) of the body forces can be expressed as

$$
\mathbf{u}^{* T} \mathbf{g}(\mathbf{x})=\mathbf{W}^{* T}(z) \hat{\mathbf{O}}(x, y) \mathbf{H}(z)
$$

where matrix $\mathbf{O}$ reads

$$
\hat{\mathbf{O}}_{k l}(x, y)=\mathbf{I}_{k l} \mathbf{V}_{k}(x, y) \mathbf{G}_{l}(x, y),
$$

with I the identity matrix.

Now, the integral results

$$
\int_{\Omega_{x y} \times \Omega_{z}} \mathbf{u}^{* T} \mathbf{g}(\mathbf{x}) d z d x d y=\int_{\Omega_{z}} \mathbf{W}^{* T}(z) \mathbf{O H}(z) d z,
$$

with

$$
\mathbf{O}=\int_{\Omega_{x y}} \hat{\mathbf{O}}(x, y) d x d y
$$

Integrating in the mesh $\Omega_{z}=\cup_{q=1}^{Q} \Omega_{z}^{q}$,

$$
\int_{\Omega_{z}} \mathbf{W}^{* T}(z) \mathbf{O H}(z) d z=\sum_{q=1}^{\mathrm{Q}} \int_{\Omega_{z}^{q}} \mathbf{W}^{q * T}(z) \mathbf{O H}^{q}(z) d z,
$$

where $\mathbf{W}^{q}(z)$ and $\mathbf{H}^{q}(z)$ are approximated respectively from

$$
\mathbf{W}^{q}(z)=\mathbf{S}(z) \mathbf{L}^{q}
$$

and

$$
\mathbf{H}^{q}(z)=\mathbf{S}(z) \mathbf{M}^{q},
$$

with $\mathbf{M}^{q}$ containing the nodal values of $\mathbf{H}(z)$ and $\mathbf{S}(z)=\left[\mathbf{N}_{1}(z) \mathbf{N}_{2}(z)\right]$, and

$$
\mathbf{N}_{i}=\left(\begin{array}{cc}
N_{i}^{q}(z) & 0 \\
0 & N_{i}^{q}(z)
\end{array}\right)
$$

Thus, it results

$$
\begin{aligned}
& \sum_{q=1}^{Q} \int_{\Omega^{q}} \mathbf{W}^{q * T}(z) \mathbf{O H}^{q}(z) d z=\sum_{q=1}^{\mathrm{Q}} \mathbf{L}^{q * T}\left\{\int_{\Omega_{z}^{q}} \mathbf{S}^{T} \mathbf{O S} d z\right\} \mathbf{M}^{q} \\
& =\sum_{q=1}^{Q} \mathbf{L}^{q * T} \mathbf{A}_{z}^{q} \mathbf{M}^{q}=\sum_{q=1}^{Q} \mathbf{L}^{q * T} \mathbf{B}_{z}^{q}=\mathbf{L}^{* T} \mathbf{B}_{z},
\end{aligned}
$$

from which, the principle of virtual works reads

$$
\mathbf{L}^{* T} \mathbf{K}_{z} \mathbf{L}=\mathbf{L}^{* T} \mathbf{B}_{z}
$$

Thus, the out-of-plane functions determining the kinematics can be obtained from a standard 1D software by using the elementary rigidity and forces given respectively by $\mathbf{K}_{z}^{q}$ and $\mathbf{B}_{z}^{q}$ considered in expression (71) and (80). 
References

1. Oñate E. Structural analysis with the finite element method. Linear statics, vol. 2., Beams, plates and shellsNew York: McGraw-Hill; 2010.

2. Bognet B, Bordeu F, Chinesta F, Leygue A, Poitou A. Advanced simulation of models defined in plate geometries: 3D solutions with 2D computational complexity. Comput Methods Appl Mech Eng. 2012;201:1-2.

3. Bognet B, Leygue A, Chinesta F. Separated representations of 3D elastic solutions in shell geometries. Adv Model Simul Eng Sci. 2014;1(1):4.

4. Quaranta G, Bognet B, Ibañez R, Tramecon A, Haug E, Chinesta F. A new hybrid explicit/implicit in-plane-out-ofplane separated representation for the solution of dynamic problems defined in plate-like domains. Comput Struct. 2018;210:135-44.

5. Owen DRJ, Hinton E. Finite elements in plasticity: theory and practice. Swansea: Pine Ridge Press; 1980.

6. Bathe KJ. Finite element procedures, vol. 2. 2006.

7. Mises RV. Mechanik der festen Krper im plastisch-deformablen Zustand. Nachrichten von der Gesellschaft der Wissenschaften zu Gottingen. Mathematisch-Physikalische Klasse. 1913;1913:582-92.

8. Siser M, Slota J. Material Model of AW 5754 H11 Al alloy for numerical simulation of deep drawing process, vol. 20. 2016.

9. Wilkins ML, Streit RD, Reaugh JE. Cumulative-strain-damage model of ductile fracture: simulation and prediction of engineering fracture tests. San Leandro: Science Applications; 1980.

\section{Publisher's Note}

Springer Nature remains neutral with regard to jurisdictional claims in published maps and institutional affiliations.

\section{Submit your manuscript to a SpringerOpen ${ }^{\circ}$} journal and benefit from:

- Convenient online submission

- Rigorous peer review

Open access: articles freely available online

- High visibility within the field

- Retaining the copyright to your article

Submit your next manuscript at $>$ springeropen.com 